\title{
Phentolamine en perfusion continue dans le traitement d'une hypertension arterielle sévère associée à un neuroblastome
}

Le traitement de l'hypertension artêrielle (HTA) sévère associée au neuroblastome est très peu discuté dans la Listérature. Chez un jeune garçon de six mois référé pour masse abdominale, on pose le diagnostic de neuroblas. rome stade IV. Ayant découvers chez lui une HTA à 26116 $\mathrm{kPa}(190 / 120 \mathrm{~mm} / \mathrm{gg})$, on décide de recourir à $u$ $\alpha$-bloquant da action rapide étant donné la malignité de la tumeur et le risque d' hémorragie intra-tumorale. Pour contrôler sa tension artérielle (TA) en phase pré-opératoire, on utilise la phentolamine 0.01 pour cent en perfusion continue titrée de $\mathrm{I}$ à $4 \mathrm{\mu g} \cdot \mathrm{kg}^{-1} \cdot \mathrm{min}^{-1}$ selon la $T A$. En même temps, on restaure le volume sanguin en utilisant comme paramètres le débit urinaire et la tension veineuse centrale. En moins de 48 heures, on peu procéder à la chirurgie. L'intervention se déroule sans incident mais seulement 80 pour cent de la tumeur peur Étre réséquée. La phentalamine cessée durant l'intervention doit être reprise de 4 à $7 \mu \mathrm{g} \cdot \mathrm{kg}^{-1} \cdot \mathrm{min}^{-1}$ en post-op suise à de nouvelles poussées hypertensives. Cinq jours plus tard, le péristaltisme ayant repris et la phénoxybenzamine p.o. étant débutée, la phentolamine est progressivement cessée en 24 heures.

L'administration de phertolamine en perfusion continue a permis un contrốle adéquat de la TA en pré-et

Départements d'anesthésie-réanimation et de pédiatrie Université de Montréal; unité des soins intensifs Hôpital Ste-Justine, Montréal.

Adresser la correspondance $\dot{a}$ : Marie Gauthier MD, Hópital Sainte-Justine, 3175 Cồte Ste-Catherine, Montréal, Canada, H3T $1 \mathrm{CS}$. post-opératoire, sans que survienne d' hypotension significative. Ce mode d"utilisation nous semble particulièrement intéressant dans le traitement de! l'HTA sévère associée au neuroblastome, où un controle rapide de la TA permet une intervention chirurgicale précoce dans des conditions optimales.

\section{Key words}

SYMPATHETIC NERVOUS SYSTEM, SYMPATHOLYTIC AGENTS: phentolamine; sURGERY: neuroblastoma.

Le neuroblastome est une tumeur maligne, résultant d'une anomalie de développement des neuroblastes qui se différencient normalement en cellules chromaffines ou ganglionnaires sympathiques. Il s'agit de la seconde tumeur maligne solide en importance chez l'enfant. Aux Etats-Unis, son incidence est approximativement de 9.6 par million chez l'enfant blanc et de 7.0 par million chez le noir.' Ses manifestations cliniques ont bien été décrites; ${ }^{2,3}$ elles varient selon le site de la tumeur primaire, la localisation des métastases et l'âge du patient. Certains signes, tels que la masse abdominale ou la fièvre inexpliqué, sont assez classiques du neuroblastome; d'autres le sont beaucoup moins comme c'est lc cas de l'hypertension artérielle dont le traitement est peu discuté dans la littérature. Nous décrivons le cas d'un enfant porteur d'un neuroblas. tome abdominal stade IV, dont l'hypertension artérielle fut traitée avec de la phentolamine en perfusion continue. 


\section{Histoire de cas}

A.P. est un jeune garçon de six mois référé à l'hôpital Sainte-Justine de Montréal le 8 janvier 1982 pour investigation d'une masse abdominale trouvée fortuitement. Dix jours plus tôt, il avait été admis dans un hốpital régional pour fièvre à $39^{\circ} \mathrm{C}$. C'est à ce moment qu'on a découvert la masse.

A l'examen physique de l'entrée, l'enfant présente une tachycardie à $140 / \mathrm{min}$., une tachypnée à $60 / \mathrm{min}$. et une température rectale à $38.7^{\circ} \mathrm{C}$. La tension artérielle n'est pas enregistrée. On note une diaphorèse profuse. L'auscultation cardio-pulmonaire est normale. A l'hypochondre droit, on palpe une masse dure, ferme, mobile latéralement, dépassant la ligne médiane et mesurant $9 \times 6 \mathrm{~cm}$. Une échographie abdominale confirme la présence d'une masse rétro-péritonéale extra-rénale et cxtrahépatique. L'urographie endovèineuse ne démontre pas de déformation importante des cavités calicielles. La série squelettique s'avère négative. A l'examen de la moelle osseuse, on décrit une infiltration d'allure néoplasique par des pseudorosettes. L'acide vanillylmandélique urinaire est à $38.2 \mathrm{mg} / 24 \mathrm{~h}$, la normale étant moins de $2 \mathrm{mg} / 24 \mathrm{~h}$ L'ensemble des données cliniques et des examens de laboratoirc cst donc compatible avec un diagnostic de neuroblastome stade IV.

L'intervention chirurgicale est retardée à cause d'une fièvre qui fluctue entre $38^{\circ}$ et $39.9^{\circ} \mathrm{C}$ et d'une tachypnée à $70 / \mathrm{min}$. Aucun foyer infectieux n'ayant pu être identifie, on conclut à une hyperthermie d'origine paranéoplasique avec tachypnée secondaire à la fièvre. On décide done d'intervenir. En salle d'opération le 13 janvier, avant l'induction de l'anesthésie, la tension artérielle est prise à $25 / 16 \mathrm{kPa}(190 / 120 \mathrm{mmHg})$. La chirurgie est annulée et le patient admis à l'unité des soins intensifs.

Après la mise en place d'un cathéter artériel et d'une tension veineuse centrale (TVC), on débute une perfusion continue de phentolamine 0.01 pour cent a $1 \mu \mathrm{g} \cdot \mathrm{kg}^{-1} \cdot \mathrm{min}^{-1}$ afin d'abaisser sa tension artérielle aux environs de $16 / 11 \mathrm{kPa}(120-130 /$ $80-85 \mathrm{mmHg}$ ) et de restaurer son volume sanguin en maintenant la TVC autour de $7 \mathrm{cmH}_{2} \mathrm{O}$. La perfusion de phentolamine est poursuivie pendant 48 heures aux environs de $4 \mu \mathrm{g} \cdot \mathrm{kg}^{-1} \cdot \mathrm{min}^{-1}$. La tension artérielle s'élève par moment à $21 / 13 \mathrm{kPa}$ $(160 / 100 \mathrm{mmHg})$, mais les poussées hypertensives répondent toujours à une augmentation temporaire de phentolamine. Le 15 janvier, le patient étant stabilisé, on procède à une laparotomie où 80 pour cent de la masse tumorale peut être réséquée. Les agents anesthésiques employés sont l'isoflurane, la métocurine et le fentanyl. La stabilité cardiovasculaire est remarquable et aucun agent vasopresseur ou anti-arythmique ne s'avère nécessaire. Le diagnostic pré-opératoire est confirmé par la pathologie.

La phentolamine, cessée durant l'intervention, doit être reprise dans la soirée du 15 janvier à cause d'une poussée hypertensive à $22 / 13 \mathrm{kPa}(170 /$ $100 \mathrm{mmHg}$ ). Une perfusion de 4 à $7 \mu \mathrm{g} \cdot \mathrm{kg}^{-1} \cdot \mathrm{min}^{-1}$ est requise dans les jours suivants pour obtenir un contrôle satisfaisant de la tension artériclle. Cinq jours après la chirurgie, le péristaltisme ayant repris, on débute la phénoxybenzamine par voie orale et on cesse la phentolamine sans problème sur une période de 24 heures. L'acide vanillylmandélique urinaire est alors mesuré à $9.6 \mathrm{mg} / 24 \mathrm{~h}$.

\section{Discussion}

La fréquence de l'hypertension artérielle associée au neuroblastome n'avait pas été bien précisée dans la littérature médicale jusqu'à tout récemment. ${ }^{4}$ Alors que plusieurs auteurs qualifiaient l'hypertension de rare ${ }^{3.5,6}$ ou n'en faisaient même pas mention, ${ }^{7}$ d'autres la décrivaient comme un problème courant. ${ }^{2,8}$ D'après l'étude rétrospective de Weinblatt et al., ${ }^{4} 1$ 'incidence de l'hypertension artérielle associée aux tumeurs neurogènes chez l'enfant serait de 19 pour cent.

Plusieurs mécanismes peuvent être invoqués quand survient une hypertension artérielle associée à un neuroblastome. La tumeur peut comprimer l'aorte ${ }^{8}$ l'artère rénale ${ }^{8,9}$ ou l'uretère. Dans ce demier cas, une hydronéphrose secondaire avec atteinte du parenchyme rénal entre en jeu. Le plus souvent, l'hypertension résulte d'une hyperproduction de catécholamines par la tumeur; elle s'accompagne occasionnellement, comme chez notre patient, de signes de stimulation sympathique tels la diaphorèse et la tachycardie. Or, l'excrétion urinaire des catécholamines et de leurs métabolites est augmentée chez la majorité des patients porteurs de neuroblastome. ${ }^{2}$ L'acide vanillylmandélique est anormalement élevé chez 75 pour cent des patients et l'acide homovanillique dans 71 pour cent des cas. ${ }^{10}$ Cette excrétion urinaire de métabolites des catécholamines, souvent augmentéc cn l'absence de signes physiologiques de relâche de noradréna- 
line, est compatible avec le fait que la noradrénaline est catabolisée à l'intérieur de la tumeur. En effet, le mécanisme de storage intra-cellulaire y est inefficace. Il en résulte une dégradation enzymatique accrue des catécholamines et une libération dans la circulation de produits pharmacologiquement inactifs. " Des études radioisotopiques auraient démontré cette inactivation des catécholamines par la cellule tumorale. ${ }^{2}$ De plus, certains neuroblastomes sécrètent un peptide intestinal vasoactif (VIP) dont les effets yasopresseurs pourraient antagoniser ceux des catécholamines. ${ }^{12}$

Le traitement de l'hypertension artérielle associée au neuroblastome ressemble à celui du phéochromocytome, cette tumeur mieux différenciée qui a une histogénèse et des propriêtés métaboliques similaires. Il s'agit cependant, dans le cas du phéochromocytome, d'une tumeur bénigne: l'urgence à intervenir y est donc moins primordiale. Plusieurs jours peuvent être alloués à la préparation pré-opératoire avec une médication administrée par voie orale. Dans le cas de A.P., étant donné la malignité de la tumeur et le risque d'hémonagie intra-tumorale, nous avons opté pour un $\alpha$-bloquant à action rapide, la phentolamine. L'administration de ce médicament en perfusion continue nous a permis de contrôler adequatement la tension artérielle en phases pré- et post-opératoire en ajustant la volémie selon la TVC et le débit urinaire. Etant donné l'absence d'arythmie ou de tachycardie significative, il n'a pas été jugé nécessaire d'ajouter un $\beta$-bloquant. Quant à l'administration de ce genre de médicament d'emblée sans diminution préalable de la résistance périphérique, elle ne fut pas envisagée étant donné l'insuffisance cardiaque qu'un $\beta$-bloquant peut causer dans de telles circonstances. Notre préparation s'est avérée satisfaisante, permettant d'éviter les problèmes per-opératoires sousjacents à cette pathologie: hypertension artérielle subite et sévère lors des manipulations de la tumeur, hypotension aiguë après le clampage veineux ou arythmies pouvant aller jusqu'à l'arrêt cardiaque, tel que l'a décrit Farman. ${ }^{13}$

Six heures environ après l'intervention, la perfusion de phentolamine a dû être reprise à cause d'une poussée hypertensive. Ceci n'est pas surprenant puisque seulement 80 pour cent de la tumeur avait pu être réséquée. Il faut mentionner qu'en phase post-opératoire de neuroblastome, on procède bien souvent à de la chimiothérapie et/ou de la radiothé- rapie, mesures qui peuvent, comme la chirurgie, faire varier subitement les niveaux circulants de catécholamines.

Bien que ce mode d'utilisation de la phentolamine n'ait été que très rarement préconisé pour le neuroblastome jusqu'à présent, ${ }^{1 / 4}$ son administration en perfusion continue est sûrement avantageuse dans un tel contexte clinique. Elle facilite en effet le contrôle de la tension artérielle autant en pré qu'en post-opératoire immédiat.

\section{References}

1 Young $J L$, Miller $R W$. Incidence of malignant tumors in U.S. children. J Pediatr 1975; 86: 254-8.

2 Fernbach DJ, Williams TE, Donaldson MH. Neurablastoma. Dans: Sutow WW, Vietti TJ, Fernbach DJ, eds. Clinical Pedjatric Oncology, Second edition. Saint-Louis: C.V. Mosby, 1977: 506-37.

3 Altman $A J$, Schwartz $A D$. Maligna ut diseases of infancy, childhood and adolescence. Philadelphia: W.B. Saunders, 1978.

4 Weinblatt ME, Heisel MA, Siegel SE. Hypertension in children with neurogenic tumors. Pediatrics 1983; 71: 947-52.

5 Evans $A E, D^{\prime}$ Angio GJ, Koop CE. Diagnosis and treatment of neuroblastoma. Pediatr Clin North Am 1976; 23: 161-70.

6 Ducket JW, Koop CE. Neuroblastoma. Urol Clin North Am 1977; 4; 285-95.

7 Grosfeld JL, Ballantine TVN, Bachner RL. Current management of childhood solid tumors. Surg Clin North Am 1976, 56: 513-35.

8 Pickett $L K$, Voorhess $M L$. Neuroblastoma in childhood. Surg Clin North Am 1964; 44: 1169-81.

9 Gianfranceschi $P$, Cambiaso P, Fasce L, Tomd P. Difficoltà nella diagnosi del neuroblastoma. Un caso con quadro clinico e bioumorale complesso con grave ipertensione arteriosa. Minerva Ped 1981; 33 : 855-60.

10 Labrosse $E H$, Com-Nougué $C$, Zucker JM, et al. Urinary excretion of 3-methoxy-4-Jiydroxymandelic acid and 3-methoxy-4-hydroxyphenylacetic acid by 288 patients with neuroblastoma and related neural crest tumors. Cancer Res 1980; 40: 1995-2001.

11 Käser H, Türler K, Wagner HP. Catecholamine metabolism in sympathetic-tumour cells. Lancet 1970; 2: 469-70.

12 Whitman JG. APUD cells and the apudomas. A concept relevant to anaesthesia and endocrinology. Anaesthesia 1977; 32: 879-88. 
13 Farman JV. Death from neuroblastoma. Br J

Anaesth 1965; 37: 883-5.

14 Tyler DC. Neuroblastoma. Dans: Stehling LC:

Common problems in pediatric anesthesia.

Chicago: Year Book Medical Publishers, 1982.

\begin{abstract}
Treatment of severe arterial hypertension associated with neuroblastoma is not well discussed in the literature. A six-month-old boy was referred for evaluation of an abdominal mass which proved to be neuroblastoma stage $I V$. Arterial hypertension of $26116 \mathrm{kPa}(19011 / \mathrm{mmHg}$ ) was also found. Because of the degree of malignancy and the risk of intra-tumoral haemorrhage, urgent manage. ment of the hypertension was required before proceeding to surgery. Phentolamine, a short-acting $\alpha$-blocking agent, was administered as a continuous infusion of a 0.01 per cent solution, at a rate of 1 to $4 \mu \mathrm{g} \cdot \mathrm{kg}^{-1} \cdot \mathrm{min}^{-1}$ titrated according to the arterial blood pressure (BP) central venous pressure and urinary output. $B P$ was rapidly controlled and the child went to surgery within 48 hours. The operation was uneventful but only 80 per cent of the tumour could be resected. Phentolamine was discontinued intraoperatively but was reinstituted post. operatively when hypertension recurred. With the return of normal intestinal function five days after surgery. phenoxybenzamine was begun p.o. and phentolamine was tapered over 24 hours and discontinued.

A continuous infusion of phentolamine provided saris. factory control pre-and post-operatively with no significant hypotension. We consider this technique to be potentially very useful in the management of severe arterial hypertension associated with neuroblastoma, as it permits early surgical intervention under optimal conditions.
\end{abstract}

